\title{
Urban travellers' mode choice: towards a new culture for urban mobility
}

\author{
E. Venezia \\ Dipartimento di scienze economiche e metodi matematici, \\ Università degli studi di Bari, Italy
}

\begin{abstract}
Rethinking urban mobility involves various aspects, among which is the optimisation of the use of all transport modes and the adoption of means of policies aimed at improving quality and accessibility of public transit networks. Nevertheless, in order to be effective, urban mobility policies need to use an approach as integrated as possible, which combines the most appropriate tools to each individual problem. To that end, it is important to have a clear knowledge of urban mobility; data collection initiatives may help to fill the gap in statistics related to the phenomenon.

In this paper, some considerations on a new culture for urban mobility are presented. In the light of new exigencies, decision-makers should support sustainable mobility-related measures to meet people's expectations.

In addition, a survey on local public transport in Bari - a southern Italian city - is presented and a random utility model has been used to investigate probable transport users' attitudes for the future. It provides indications on the needs of public transport users and there are lessons that can be learned from the Bari case.
\end{abstract}

Keywords: urban transport demand, users' choice, random utility model.

\section{European framework}

Sustainable transport is crucial for a higher quality of life in European urban areas and enables the reduction of congestion, pollution and accidents. First of all, a greater awareness for all travellers about the impact of their mobility choices is an essential element towards the new culture of urban mobility [1]. Secondly, in order to promote alternatives to cars, the availability of public transport as a complementary mode is very important. 
The European Commission is suggesting the use of push and pull measures, which could be successfully applied, if coordinated and introduced at the same time as a mixed policy. Experience in Member States shows that pull measures are tools making public transport more attractive, while push measures use deterring or punishing tools to promote modal shift. The combination of these two different tools is the winning solution.

What is the current public transport situation in Europe?

In accordance with UITP [2], some key messages can be considered:

- urban areas generate $75 \%$ to $85 \%$ of Gross National Product

- $\quad$ around $80 \%$ of Europe's population live in urban areas

- cars account for $75 \%$ of urban kilometres travelled

- congestion costs have a serious impact on business competitiveness

- every year 4.3 million extra cars take to the road in Europe

- 890 billion passenger kilometres travelled on public transport accounts for the equivalent of 44 million cars

- a $1 \%$ shift from cars to public transport would reduce traffic by the equivalent of 2 million cars

- $\quad$ EUR 1 million invested in public transport infrastructure generates EUR 2 to 2.5 million additional local Gross Domestic Product and 30 jobs

- good access to local public transport is a key factor in business location.

In 2006, ntra-EU-27 passenger transport and domestic transport demand using passenger cars, powered two-wheelers, buses, coaches and railways, as well as trams and the metro, was 5,746 billion pkm or 11,674 $\mathrm{km}$ per person. In addition, passenger cars accounted for $80.1 \%$ of this total, powered two-wheelers for $2.7 \%$, buses and coaches for $9.1 \%$, railways for $6.7 \%$ and trams and the metro for $1.5 \%$. The unbalanced situation is, therefore, clear.

But how can collective transport be attractive for transport users? In different ways. These are some examples:

- $\quad$ good information for travellers such as, for example, timetables and fares

- $\quad$ good quality of service, which means, for instance, improving service frequency and travel speed

- pleasant and comfortable journey

- $\quad$ attractive intermodal nodes

- $\quad$ easy accessibility to the service (in terms of means and stops).

Therefore, the idea is that it is preferable, in terms of time, is to use these "soft" measures and to work on a change of personal mobility choices instead of waiting to renew a national vehicle fleet (which may take 20 years) or implementing new technologies. Without major improvement in public transport efficiency, public transport will not be able to compete with private car transport.

In order to do so, customer focus is an essential element in the development of high quality public transport that may better meet customers' needs. In order to deliver more tailor-made yet efficient public transport services, travel patterns need to be analysed in as much detail as possible. 
Therefore, analysing travel behaviour can tell us something more about likely future levels of bus use and, particularly, factors affecting travel demand. In addition, survey results shed some light on present problems linked to the currently supplied transport service.

\section{Survey results and empirical analysis}

In the next section, focus will be placed on the results related to all public transport users. They have been extrapolated from an interview survey which accounted for a total of 1,198 respondents (users and non users) - of all age groups - and was conducted in Bari in 2008. A non-probability sampling technique was adopted and respondents were approached in the city centre area and in peripheral areas. The time of the day was 7.00-21.00.

The questionnaire was designed and formulated to explore individual relations of all transport users to buses. The objective of the survey was to fill the big gap in urban mobility statistics and to provide the necessary information to decision-makers and practitioners at all levels. Survey results and empirical analysis could also help general public with necessary data and improve knowledge on urban mobility. The topics that will be presented in this paper pertain to users' behaviour and evaluate the probability of those using buses, according to some characteristics.

\subsection{Mobility characteristics of public transport users}

This part of the sample has 647 respondents, split in $48 \%$ males and $52 \%$ females. The age composition of this sample is structured with $37.5 \%$ users in the $19-29$ year age range, followed by $24.5 \%$ in the $30-50$ range, while $7.5 \%$ are over $65,17 \%$ are in the 51-65 range and $13.5 \%$ is represented by users under 19 .

As for the occupational composition of the sample, students account for $38 \%$ of the respondents, housewives for $14 \%$ and retirees for $15 \%$. Only $33 \%$ of total passengers are employed, mainly employees (24\%), while the self-employed are only $9 \%$.

Results point out that interviewees, who use public transport, are those who have no cars or other transport means. As a matter of fact, out of the total number of public transport users included in this sample, $49 \%$ of total respondents have always private means for all day. Only $36 \%$ of public transport users have sometimes private means, while the remaining $15 \%$ can use alternatives only occasionally. Furthermore, $23 \%$ of the respondents use buses daily and $75 \%$ of the total purchase tickets daily.

Most passengers (73\%), although owning a car, prefer using a bus, which they consider comfortable, as it avoids the waste of time caused when looking for a parking place. This aspect is so relevant that it causes bus delay. Indeed, $42 \%$ of the sample is not satisfied by bus frequency and they ask for service intensification. Many users desire security enforcement on board: $33 \%$ of passengers are afraid of road accidents, pickpockets and violence on board. Punctuality, frequency and safety could be obtained with a higher level of 
operator investments. Users declared that they would be willing to pay something more in exchange for compliance with their requests. In particular, $68 \%$ users would pay something more for a higher frequency. As for equity bus service price, $95 \%$ of respondents are in favour.

Finally, results show that interviewees are still poorly sensitive to pollution problems and scarcely informed on new tools for improving traffic conditions: only $17 \%$ are aware of car pooling, collective taxis and car sharing tools. Furthermore, $68 \%$ of these respondents would use these alternative transport modes, and $61 \%$ would be in favour of a substitution of private cars with new means of transport.

\subsection{Empirical analysis}

The aim of this section is to reveal quantitatively the probability of people using public transport offered in Bari. Results stem from the application of a random utility model.

To give a further interpretation of the data on individual choice related to the bus service provided in Bari, the random utility model has been used.

As indicated by Green [3], suppose that $y_{m}$ and $y_{p}$ represent the individual's utility of two choices, denoted $\mathrm{U}^{\mathrm{a}}$ e $\mathrm{U}^{\mathrm{b}}$. The observed choice between the two reveals which one provides the greater utility. Therefore, the observed indicator equals 1 if $\mathrm{Ua}>\mathrm{U}^{\mathrm{b}}$ and 0 if $\mathrm{Ua} \leq \mathrm{U}^{\mathrm{b}}$. A common formulation of the linear random utility model is:

$\mathrm{U}^{\mathrm{a}}=\beta^{\prime}{ }_{\mathrm{a}} \mathrm{x}+\varepsilon_{\mathrm{a}}$ and $\mathrm{U}^{\mathrm{b}}=\beta^{\prime}{ }_{\mathrm{b}} \mathrm{x}+\varepsilon_{\mathrm{b}}$. Then if we denote by $\mathrm{Y}=1$ the consumer's choice of alternative a, we have:

$$
\begin{aligned}
\operatorname{Prob}[\mathrm{Y}=1 \mid \mathbf{x}] & =\operatorname{Prob}\left[\mathrm{U}^{\mathrm{a}}>\mathrm{U}^{\mathrm{b}}\right] \\
& =\operatorname{Prob}\left[\beta^{\prime}{ }_{\mathrm{a}} \mathbf{x}+\varepsilon_{\mathrm{a}}-\beta^{\prime}{ }_{\mathrm{b}} \mathbf{x}-\varepsilon_{\mathrm{b}}>0 \mid \mathbf{x}\right] \\
& =\operatorname{Prob}\left[\left(\beta_{\mathrm{a}}-\beta_{\mathrm{b}}\right)^{\prime} \mathbf{x}+\varepsilon_{\mathrm{a}}-\varepsilon_{\mathrm{b}}>0 \mid \mathbf{x}\right] \\
& =\operatorname{Prob}\left[\beta^{\prime} \mathbf{x}+\varepsilon>0 \mid \mathbf{x}\right] .
\end{aligned}
$$

The individual's utility of two choices - bus transit and private means - is estimated by binary logistic regression and logistic regression coefficients are used to estimate odds ratios for each independent variable in the model. The value assumed by the dependent variable, as the probability to use buses, is equal to 1 . All the values assumed by independent variables have been transformed into dummy variables in order to capture each characteristic of independent variables represented by sex, occupational status and so on. Equations have been estimated by using single attributes to avoid evident correlation problems and a consequent self-selectivity involved in the data. Here, the selection is given by the significance of parameters, which has been checked with the Wald statistic at a 5\% level. All parameters have been chosen with the Wald forward selection method and values reported in Table 1 are all significant in accordance with the Wald test.

Table 1 shows values assumed by coefficients as odds ratios. They indicate the probability of using a public bus for each characteristic, against the probability of using other means in an urban context. 
Table 1: $\quad$ Probability of bus use.

\begin{tabular}{lll}
\hline Age & $19-29$ & Public buses \\
& $30-50$ & 9.81 \\
& $51-65$ & 4.99 \\
Availability of other & & 13.12 \\
means & & \\
& Always & \\
Frequency in the bus use & Sometimes & 2.72 \\
& & 1.07 \\
& Every day & \\
Reasons & 1-2 times per week & 93.29 \\
& Rarely & 4.52 \\
& & 1.02 \\
Occupational profile & School/Working activity & 12.51 \\
& Leisure activity & 8.66 \\
& Shopping & 5.47 \\
& & \\
& Student & 12.06 \\
& Self employed & 6.75 \\
& Housewife & 3.12 \\
& Employee & 12.70 \\
& Retiree & 10.69 \\
\hline
\end{tabular}

Figures show that the probability of using buses is particularly important for those belonging to the 51-65 year-old range, followed by those who are between 19-29, and those in the 30-50 year-old range. For those who always have a car or other transport means, the probability of using buses is more than twice as much as those who sometimes have a car.

The most important reasons that may influence people to get a bus are studying or working: these motivations are three times more important than reasons related to shopping and one and a half times more important than reasons related to leisure activities. Finally, as to the occupational profile, employees and students are four times keener on using buses than housewives. This can be explained by the fact that the first two components' demand is systematic, whereas housewives' may be erratic.

\section{Lessons from Bari}

Aspects affecting attitudes are usually classified as: behavioural, affective and cognitive [4]. Considering these aspects in the analysis can surely increase the predictive ability of a model by a clearer specification of the relationship between attitudes and behaviour. This relation, improved with some social elements, gave way to the theory of planned behaviour elaborated by Ajzen in 1991 and has been subsequently applied to transport research especially with regard to environmental problems [5]. These studies drew the conclusion that the choice of transport modes is largely a reasoned decision related particularly to 
attitudes. Other authors, on the other side, suggest including independent measures of habit to further improve the predictive capability of attitudebehaviour studies [6].

More generally, Recker and Golob [7] in their paper found that attitudinal data may be better predictors of modal choice than the traditional objective measures, such as travel time and cost. With regard to this approach, Recker and Stevens [8] considered some attributes of relevance for shopping activities. Some of this attributes are still valid although, as the results of the questionnaire presented here indicate, some elements have a different burden in the modal choice of bus service in comparison with alternative modes.

Results suggest that policy makers and transport operators should consider at least the profiles of two demand components (systematic and erratic) to intervene in the urban system to achieve some positive results in terms of sustainability. The possibility of using buses is greatly affected by the age structure of respondents, by the reason why people use buses instead of private means and by the occupational profile. Considering these elements may fill the gap existing between the demand and supply of bus services in an urban context, and consequently may shift car users to buses. Another important element is given by the fact that a large part of interviewees would even pay more to get better service quality, which means that there is the willingness to this bus shift. Therefore, in accordance with White [9], it is advisable to have:

- greater affordable and stable service

- comprehensive information for users.

The very comprehensive nature of statistics available in Bari may be matched elsewhere and the introduction of stable and well structured data collection in those areas where new policies may be tried is essential for effective monitoring to take place.

Still, several issues have been addressed in the present study and available data can be considered for future research, particularly the remaining part of the survey referred to those who currently do not use buses. Thus, further research is needed to reinforce the validity of findings in this study and to promote transportation policies in the direction of a change in travel behaviour.

\section{Acknowledgement}

This paper is part of the research project "Impatto dell'integrazione tariffaria sulla domanda di trasporto pubblico locale", funded by the University of Bari.

\section{References}

[1] Commission of the European Communities, Green paper, Towards a new culture for urban mobility, COM (2007) 551 final, 2007.

[2] UITP, European Commission, Public transport, the Lisbon Strategy and Sustainable Development, position paper, July, 2005.

[3] Green, W.H., Econometric analysis, Prentice-Hall International, London, pp. 871-901, 1997. 
[4] Eagley, A., Chaiken, S., The psychology of attitudes, Harcourt Brace Jovanovich College Publishers, New York, 2003.

[5] Kaiser, F., Wölfing, S., Fuhrer, U. (1999), Environmental attitude and ecological behaviour, Journal of Environmental Psychology, 19, pp.1-19.

[6] ANBLE, J. (2005), "Complacent Car Addicts' or 'Aspiring Environmentalists'? Identifying travel behaviour segments using attitude theory, Transport Policy, 12, pp. 65-78.

[7] Recker, W., Golob, T.F., "An attitudinal modal choice model", Transportation Research, 10, pp. 299-310, 1976.

[8] Recker, W.-Stevens, R., "Attitudinal models of modal choice: the multinomial case for selected nonwork trips", Transportation, 3, pp. 343356, 1976.

[9] White, P. R., "Factors Affecting the Decline of Bus Use in the Metropolitan Areas", Report, Passenger Transport Executive Group, 2008. 